\title{
Experimental Study of a Person Identification Framework Based on Combining Left and Right Palmprint Images
}

\author{
Mane Bhagyashri ${ }^{1}$, Kalyankar Pravin ${ }^{2}$ \\ T.P.C.T.'s College Of Engineering, Osmanabad, Solapur-Osmanabad Road, Osmanabad, India
}

\begin{abstract}
Multibiometrics can provide higher identification accuracy than single biometrics, so it is more suitable for some real-world personal identification applications that need high-standard security. Among various biometrics technologies, palmprint identification has received much attention because of its good performance. Combining the left and right palmprint images to perform multibiometrics is easy to implement and can obtain better results. However, previous studies did not explore this issue in depth. In this paper, we proposed a novel framework to perform multibiometrics by comprehensively combining the left and right palmprint images. This framework integrated three kinds of scores generated from the left and right palmprint images to perform matching score-level fusion. The first two kinds of scores were, respectively, generated from the left and right palmprint images and can be obtained by any palmprint identification method, whereas the third kind of score was obtained using a specialized algorithm proposed in this paper. As the proposed algorithm carefully takes the nature of the left and right palmprint images into account, it can properly exploit the similarity of the left and right palmprints of the same subject. Moreover, the proposed weighted fusion scheme allowed perfect identification performance to be obtained in comparison with previous palmprint identification methods.
\end{abstract}

Keywords: biometrics, multibiometrics, MFRAT, palmprint.

\section{Introduction}

Palmprint identification is an important personal identification technology and it has attracted much attention. The palmprint contains not only principle curves and wrinkles but also rich texture and miniscule points, so the palmprint identification is able to achieve a high accuracy because of available rich information in palmprint.

Various palmprint identification methods, such as coding based methods and principle curve methods have been proposed in past decades. In addition to these methods, subspace based methods can also perform well for palmprint identification. For example, Eigenpalm and Fisherpalm are two well-known subspace based palmprint identification methods.

In recent years, 2D appearance based methods such as 2D Principal Component Analysis (2DPCA), 2D Linear Discriminant Analysis (2DLDA), and 2D Locality Preserving Projection (2DLPP) have also been used for palmprint recognition. Further, the Representation Based Classification (RBC) method also shows good performance in palmprint identification. Additionally, the Scale Invariant Feature Transform (SIFT), which transforms image data into scaleinvariant coordinates, are successfully introduced for the contactless palmprint identification. No single biometric technique can meet all requirements in circumstances . To overcome the limitation of the unimodal biometric technique and to improve the performance of the biometric system, multimodal biometric methods are designed by using multiple biometrics or using multiple modals of the same biometric trait, which can be fused at four levels: image (sensor) level, feature level, matching score level and decision level.

\section{Literature Survey}

Huanga, Jiaa and Zhang have proposed the palmprint authentication system based on principal line extraction. Modified finite Radon transform has been employed for feature extraction. A line matching technique has been used which is based on the pixelto- area algorithm Type fonts are required. Please embed all fonts, in particular symbol fonts, as well, for math, etc. Zhang, Kong, You and Wong had proposed Online Palmprint Identification. We proposed scheme takes online palmprints, and utilizes low resolution images. Low pass filter in addition to boundary tracking algorithm is used in the pre-processing phase. A normalized hamming distance is used for matching.

Anil K Jain, ArunRose proposed Multibiometric systems which describes about the limitations imposed by unimodal systems can be solves by using multimodal systems. Yong $\mathrm{Xu}$, Lunke Fei, and David Zhang proposed the paper Combining Left and Right Palmprint Images for More Accurate Personal Identification. We proposed framework shows that the left and right palmprint images of the same subject are somewhat similar. We proposed method carefully takes the nature of the left and right palmprint images into account, and designs an algorithm to evaluate the similarity between them. Moreover, by employing this similarity, the proposed weighted fusion scheme uses a method to integrate the three kinds of scores generated from the left and right palmprint images.

\section{The Proposed Framework}

\subsection{Overview of the technique}

There are different types of palmprint technique which are used for identifying accurate personal. 


\section{International Journal of Science and Research (IJSR) \\ ISSN (Online): 2319-7064}

Index Copernicus Value (2015): 78.96 | Impact Factor (2015): 6.391

- PalmPrint Identification Across Left Palm

- PalmPrint Identification Across Right Palm

- PalmPrint Identification Across Crossing Palm

- PlamPrint Identification Across Combining Left, Right and Crossing PalmPrint

\section{PalmPrint Identification across Left Palm:}

The framework first works for the left palmprint images and uses a palmprint identification method to calculate the scores of the test sample with respect to each class. Consider $\mathrm{m}$ available left palmprint images for training.Let $\mathrm{X}_{\mathrm{i}}^{\mathrm{k}}$ denote the $i$ th left palmprint images of the $k$ th subject, where $i=1 \ldots . m$ and $\mathrm{k}=1 \ldots \mathrm{c}$. Let $\mathrm{Z} 1$ stand for left palmprint image [test sample].Then PalmPrint Principal Lines extraction performed by Modified Finite Radon Transform. After the extract of principal lines such as left palmprint principal lines and left training palmprint principal lines, then matching score is calculated.

\section{PalmPrint Identification across Right Palm:}

The palmprint identification method to the right palmprint images to calculate the score of the test sample with respect to each class. Consider $\mathrm{m}$ available right palmprint images for training. Let $\mathrm{Y}_{\mathrm{i}}^{\mathrm{k}}$ denote the $\mathrm{i}$ th right palmprint images of the $\mathrm{k}$ th subject, where $\mathrm{i}=1 \ldots \mathrm{m}$ and $\mathrm{k}=1$...c. Let $\mathrm{Z} 2$ stand for right palmprint image [test sample].Then PalmPrint Principal Lines extraction performed by Modified Finite Radon Transform. After the extract of principal lines such as right palmprint principal lines and right training palmprint principal lines, then matching score is calculated.

\section{PalmPrint Identification across Crossing Palm:}

Crossing matching score of the left palmprint image for testing with respect to the reverse right palmprint images of each class is obtained.Generate the reverse images $\tilde{Y}_{i}^{k}$ of the right palmprint images $\mathrm{Y}_{\mathrm{i}}^{\mathrm{k}}$.Both $\mathrm{Y}_{\mathrm{i}}^{\mathrm{k}}$ and $\tilde{Y}_{i}^{k}$ will be used training sample.

$\tilde{Y}_{i}^{k}$ is obtained by: $\tilde{Y}_{i}^{k}(i, c)=Y_{i}^{k}\left(L_{Y}-l+1, c\right)$, $(\mathrm{l}=1 \ldots \mathrm{Ly}, \mathrm{c}=1 \ldots \mathrm{Cy})$, where Ly and $\mathrm{Cy}$ are the row number and column number of $\mathrm{Y}_{\mathrm{i}}^{\mathrm{k}}$ respectively. Then PalmPrint Principal Lines extraction performed by Modified Finite Radon Transform. After the extract of principal lines such as left palmprint principal lines and reverse right training palmprint principal lines, then matching score is calculated.

\section{PalmPrint Identification across Left, Right and Crossing PalmPrint:}

Finally the framework performs matching score level fusion to integrate these three scores such as left matching score, right matching score and crossing matching score to obtain the identification result. wi(i $=1,2,3)$, which denotes the weight assigned to the ith matcher, can be adjusted and viewed as the importance of the corresponding matchers.

\subsection{Similarity between the Left and Right Palmprints}

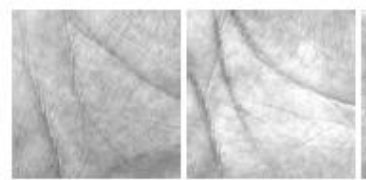

(a)

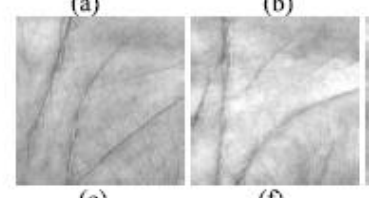

(e)

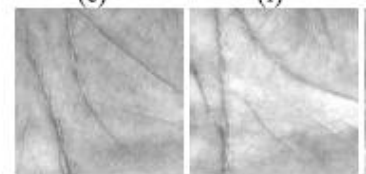

(i) (j)



(g)

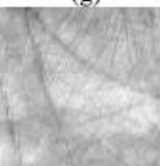

(k)
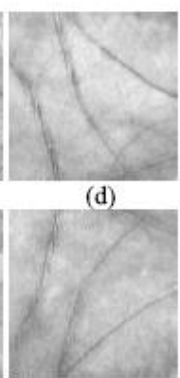

(h)
Figure 1: Palmprint images of four subjects. (a)-(d) are four left palmprint images; (e)-(h) are four right palmprint corresponding to (a)-(d); (i)-(l) are the reverse right palmprint images of (e)-(h)
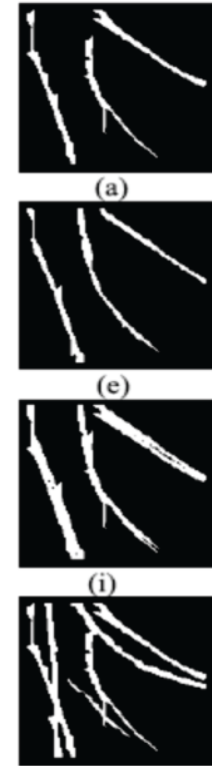

(m)
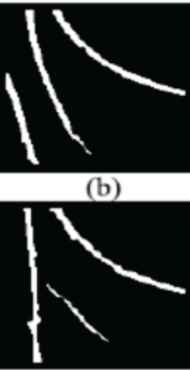

(f)

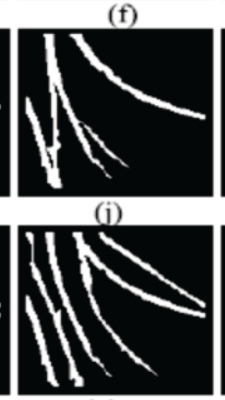

(n)

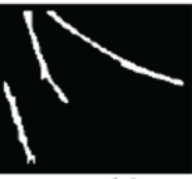

(c)

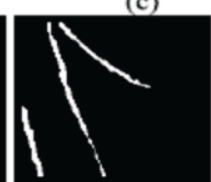

(g)

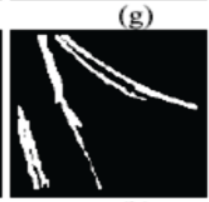

(k)

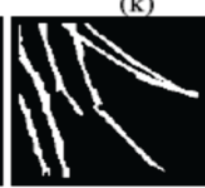

(o)
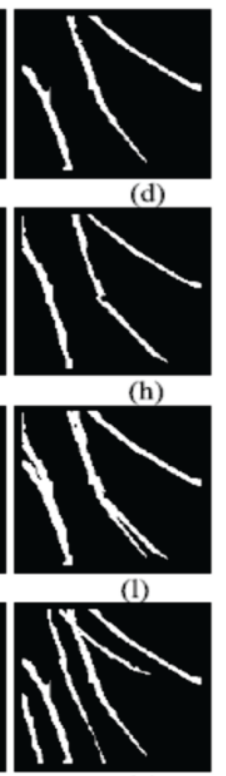

(p)

Images in Figure 1 (i)-(l) are the four reverse palmprint images of those shown in Figure 1 (e)-(h). It can be seen that the left palmprint image and the reverse right palmprint image of the same subject are somewhat similar.

Figure 2 (a)-(d) depicts the principal lines images of the left palmprint shown in Figure 1 (a)-(d). Figure 2 (e)-(h) are the reverse right palmprint principal lines images corresponding to Figure 1 (i)-(1). Figure 2 (i)-(1) show the principle lines matching images of Figure 2 (a)-(d) and Figure 2 (e)-(h), respectively. Figure $2(\mathrm{~m})-(\mathrm{p})$ are matching images between the left and reverse right palmprint principal lines images from different subjects. The four matching images of Figure 1 (m)-(p) are: (a) and ( f) principal lines matching image, (b) and (e) principal lines matching image, (c) and (h) principal lines matching image, and (d) and (g) principal lines matching image, respectively.

Figure 2 (i)-(1) clearly show that principal lines of the left and reverse right palmprint from the same subject have very

\section{Volume 6 Issue 7, July 2017}




\section{International Journal of Science and Research (IJSR) \\ ISSN (Online): 2319-7064}

Index Copernicus Value (2015): 78.96 | Impact Factor (2015): 6.391

similar shape and position. However, principal lines of the left and right palmprint from different individuals have very different shape and position, as shown in Figure 2 (m)-(p). It is demonstrates that the principal lines of the left palmprint and reverse right palmprint can also be used for palmprint verification/identification.

Figure 2. Principal lines images. (a)-(d) are four left palmprint principal lines images, (e)-(h) are four reverse right palmprint principal lines image, (i)-(l) are principal lines matching images of the same people, and (m)-(p) are principal lines matching images from different people.

\subsection{Principle Line Extraction using Modified Finite}

\section{Random Transform}

In our scheme, the first step is to extract principal lines. We have proposed a modified finite radon transform (MFRAT) to extract principal lines, and in our project the MFRAT will also be adopted to detect some key points of principal lines for fast retrieval. Here, the definition of MFRAT is given as follows: Denoting $\mathrm{Zp}=\{0,1, \ldots, \mathrm{p}-1\}$, where $\mathrm{p}$ is a positive integer, the MFRAT of real function $\mathrm{f}[\mathrm{x}, \mathrm{y}]$ on the finite grid $\mathrm{Z}^{2}$ is defined as:

$$
r\left[L_{k}\right]=\operatorname{MFRAT}_{\gamma}(k)=\frac{1}{S_{(i, j) \in L_{k}}} \sum_{f} f[i, j]
$$

where $\mathrm{S}$ is a scalar to control the scale of $\mathrm{r}\left[\mathrm{L}_{\mathrm{k}}\right]$, and $\mathrm{L}_{k}$ denotes the set of points that make up a line on the lattice $\mathrm{Z}^{2}$ which means:

$$
L_{l}=\left\{(i, j): j=k\left(i-i_{i}\right)+j_{j i} i \in L_{p}\right\}
$$

where $\left(i_{0}, j_{0}\right)$ denotes the center point of the lattice $\mathrm{Z}_{\mathrm{p}}^{2}$, and $\mathrm{k}$ means the corresponding slope of $\mathrm{L}_{\mathrm{k}}$. In our project, $\mathrm{L}_{\mathrm{k}}$ has another expression i.e. $\mathrm{L}(\theta \mathrm{k})$, where $\theta \mathrm{kis}$ the angle corresponding to different index $\mathrm{k}$. In the MFRAT, the direction $\theta \mathrm{k}$ and the energy e of center point $\mathrm{f}\left(\mathrm{i}_{0}, \mathrm{j}_{0}\right)$ of the lattice $\mathrm{Z}_{\mathrm{p}}^{2}$ are calculated by following formula:

$$
\begin{gathered}
\theta_{k\left(i_{0}, j_{0}\right)}=\arg \left(\min _{k}\left(r\left[L_{k}\right]\right)\right) \quad k=1,2, \cdots N \\
e_{\left(i_{0}, j_{0}\right)}=\left|\min \left(r\left[L_{k}\right]\right)\right| \quad k=1,2, \cdots N
\end{gathered}
$$

Where $|\cdot|$ means the absolute operation. In this way, the directions and energies of all pixels can be calculated if the center of lattice $\mathrm{Z}^{2}$ p moves over an image pixel by pixel. And then, two new images i.e. Direction image and Energy image, will be created, which can be used for subsequent extraction of principal lines.

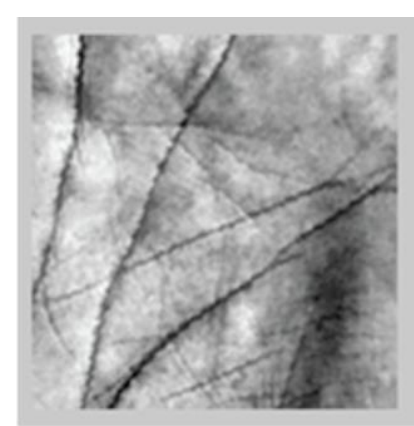

(a)

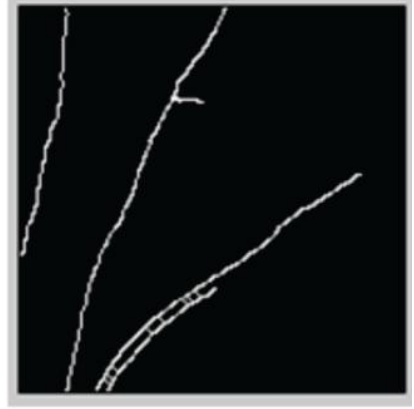

(b)
Figure 3: (a) ROI of left palmprint image given to modified finite random transform (b) Image after applying canny edge detector

\section{Procedure of the Proposed Framework}

The framework first works for the left palmprint images and uses a palmprint identification method to calculate the scores of the test sample with respect to each class. Then it applies the palmprint identification method to the right palmprint images to calculate the score of the test sample with respect to each class. After the crossing matching score of the left palmprint image for testing with respect to the reverse right palmprint images of each class is obtained, the proposed framework performs matching score level fusion to integrate these three scores to obtain the identification result.

We suppose that there are $C$ subjects, each of which has $m$ available left palmprint images and $m$ available right palmprint images for training. Let $X k i$ and $Y k i$ denote the $i$ th left palmprint image and $i$ th right palmprint image of the $k$ th subject respectively, where $i=1, \ldots, m$ and $k=1, \ldots, C$. Let $Z 1$ and $Z 2$ stand for a left palmprint image and the corresponding right palmprint image of the subject to be identified. $Z 1$ and $Z 2$ are the so-called test samples.

Step 1: Generate the reverse images $Y^{r} i$ of the right palmprint images $Y k i$. Both $Y k i$ and $Y k i$ will be used as training samples. $Y^{\sim} k i$ is obtained by: $Y^{\sim} k i(l, c)=Y k i(L Y$ $-l+1, c),(l=1 \ldots L Y, c=1 \ldots C Y)$, where $L Y$ and $C Y$ are the row number and column number of $Y k i$ respectively.

Step 2: Use $Z 1, X_{k} i$ s and a palmprint identification method, such as the method introduced in Section II, to calculate the score of $Z_{1}$ with respect to each class. The score of $Z_{1}$ with respect to the $i$ th class is denoted by $s i$.

Step 3: Use $Z_{2}, Y_{k} i$ s and the palmprint identification method used in Step 2 to calculate the score of $Z_{2}$ with respect to each class. The score of $Z_{2}$ with respect to the $i$ th class is denoted by $t i$.

Step 4: $Y^{\sim} k j\left(j=1, \ldots, m_{-}, m_{-} \leq m\right)$, which have the property of Sim_score( $\sim Y k j, X k) \geq$ match_threshold, are selected from $Y^{\sim} k$ as additional training samples, where match_threshold is a threshold. Sim_score ( $Y k j, X k)$ is defined as: 


\section{International Journal of Science and Research (IJSR) \\ ISSN (Online): 2319-7064}

Index Copernicus Value (2015): 78.96 Impact Factor (2015): 6.391

$$
\operatorname{Sim} \_\operatorname{score}\left(Y, X^{k}\right)=\sum_{t=1}^{T}\left(S\left(\hat{Y}, X^{k}\right)\right) / T
$$

and

$$
S\left(\hat{Y}, X^{k}\right)=\max \left(\operatorname{Score}\left(\underset{t}{\hat{Y}}, \hat{X}_{i}^{k}\right)\right), \quad i=\{1 \ldots m\}
$$

where $Y$ is a palmprint image. $X k$ are a set of palmprint images from the $k$ th subject and $X k i$ is one image from $X k$. ${ }^{\wedge} X k i$ and $Y$ are the principal line images of $X k i$ and $Y$, respectively. $T$ is the number of principal linesof the palmprint and $t$ represent the $t$ th principal line. $\operatorname{Score}(Y, X)$ is calculated as formula (1) and the $\operatorname{Score}(Y, X)$ is set to 0 when it is smaller than sim_threshold, which is empirically set to 0.15 .

Step 5: Treat $Y^{r} k j$ s obtained in Step 4 as the training samples of $Z 1$. Use the palmprint identification method used in Step 2 to calculate the score of $Z 1$ with respect to each class. The score of the test sample with respect to $Y^{r} k j$ s of the $i$ th class is denoted as $g i$.

Step 6: The weighted fusion scheme $f i=w 1 s i+w 2 t i+w 3 g i$, where $0 \leq w 1, w 2 \leq 1$ and $w 3=1-w 1-w 2$, is used to calculate the score of $Z 1$ with respect to the $i$ th class. If $q=$ $\operatorname{argmin} I f i$, then the test sample is recognized as the $q$ th subject.



Figure 4: Fusion at the matching score level of the proposed framework

\section{Result}

In the proposed method, since the processing of the reverse right training palm print can be performed before palm print identification, the main computational cost of the proposed method largely relies on the individual palm print identification method. Compared to the conventional fusion strategy those only fuses two individual matchers, the proposed method consists of three individual matches. As a result, the proposed method needs to perform one more identification than the conventional strategy. Thus, the identification time of the proposed method may be reduced, compared to conventional fusion strategy. The output screenshots of the proposed system as shown in following figures.
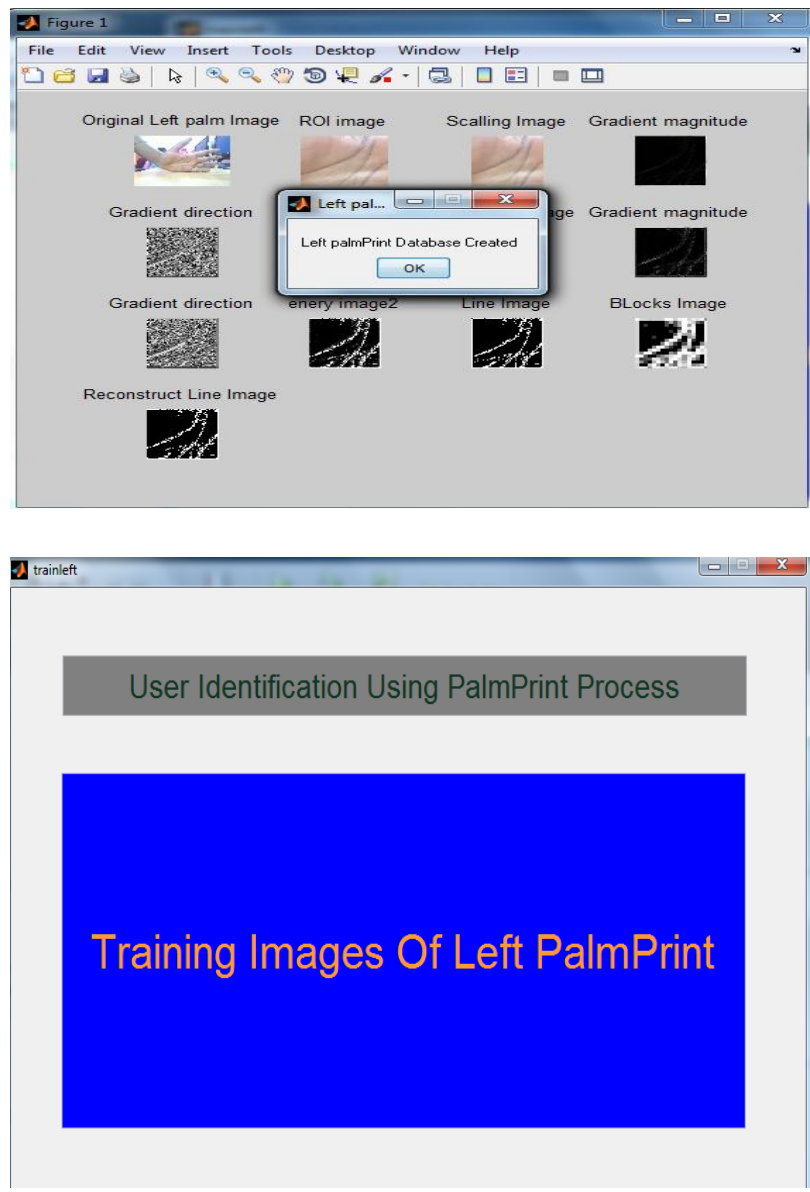

Figure 5: Left palmprint image

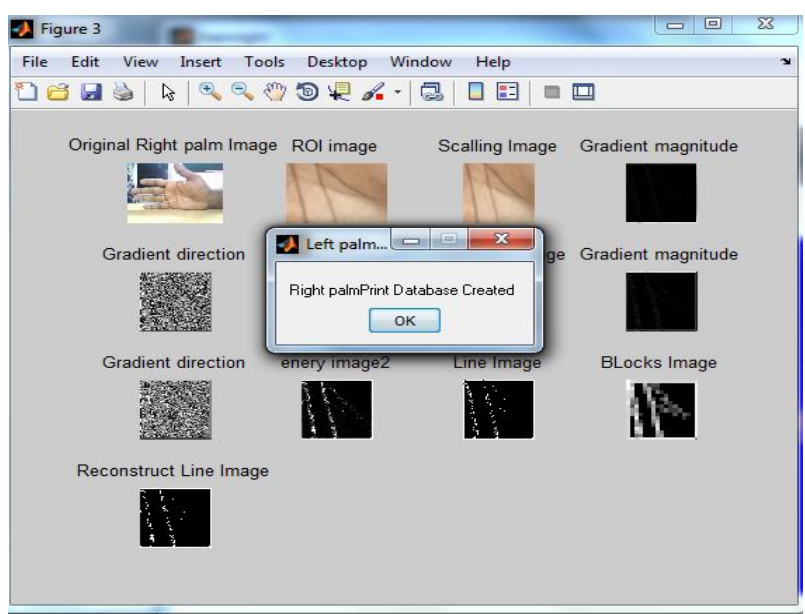




\section{International Journal of Science and Research (IJSR) \\ ISSN (Online): 2319-7064}

Index Copernicus Value (2015): 78.96 | Impact Factor (2015): 6.391

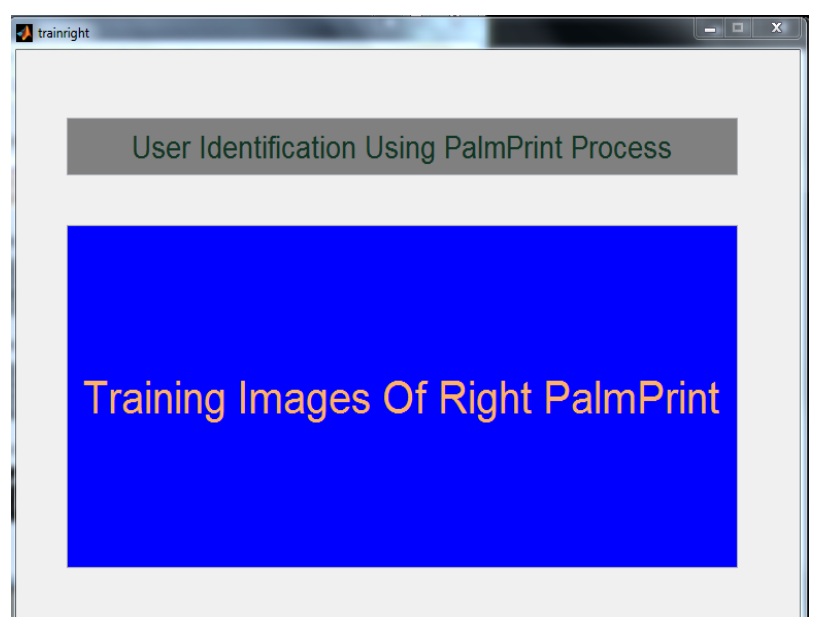

Figure 6: Right palmprint image


Figure 7: Reverse right palmprint image


Figure 8: Describes the matching between two palm images

\section{Conclusions}

In this paper we demonstrated that the left and right palm print images of the same subject are almost similar. For the performance improvement of palm print identification by using the similar patterns has been proposed in this paper. The proposed method carefully takes the nature of the left and right palm print images into account, and designs an algorithm to evaluate the similarity between them as we used modified finite random transform techniques. Since, by utilizing this similarity, the proposed weighted fusion scheme uses a method to integrate the three kinds of scores generated from the left and right palm print images. Effective experimental results shows that the proposed framework obtains very high accuracy as we used many pre-processing techniques and the use of the similarity score between the left and right palm print leads to important improvement in the accuracy. This work also seems to be helpful in motivating people to explore potential relation between the traits of other bimodal biometrics issues.

\section{References}

[1] D. S. Huang, W. Jia, and D. Zhang, "Palmprint verification based on principal lines, " Pattern Recognition., vol. 41, no. 4, pp. 1316-1328, Apr. 2008 
[2] A. W. K. Kong, D. Zhang, and M. S. Kamel, "A survey of palmprint recognition," Pattern Recognit., vol. 42, no. 7, pp. 1408-1418, Jul. 2009.

[3] D. Zhang, F. Song, Y. Xu, and Z. Lang, “Advanced pattern recognition technologies with applications to biometrics, ” Med. Inf. Sci. Ref., Jan. 2009, pp. 1-384.

[4] R. Chu, S. Liao, Y. Han, Z. Sun, S. Z. Li, and T. Tan, "Fusion of face and palmprint for personal identification based on ordinal features, " in Proc. IEEE Conf. Comput. Vis. Pattern Recognit. (CVPR), Jun. 2007, pp. $1-2$.

[5] Z. Sun, T. Tan, Y. Wang, and S. Z. Li, "Ordinal palmprint represention for personal identification [represention read representation]," in Proc. IEEE Conf. Comput. Vis. Pattern Recognit., vol. 1. Jun. 2005, pp. 279-284.

[6] A. Kong, D. Zhang, and M. Kamel, "Palmprint identification using feature-level fusion, " Pattern Recognit., vol. 39, no. 3, pp. 478-487, Mar. 2006.

[7] D. Zhang, W.-K. Kong, J. You, and M. Wong, "Online palmprint identification," IEEE Trans. Pattern Anal. Mach. Intell., vol. 25, no. 9, pp. 1041-1050, Sep. 2003.

[8] A. Morales, M. A. Ferrer, and A. Kumar, "Towards contactless palmprint authentication," IET Comput. Vis., vol. 5, no. 6, pp. 407-416, Nov. 2011.

\section{Author Profile}

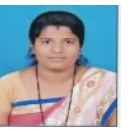

Ms. Bhagyashri Kamlakar Mane, is a M.E student of Computer Science \& Engineering from T.P.C.T.'s College of Engineering, Osmanabad India. She graduated in Computer Science and Engineering from BAMU University, Aurangabad. Her current research work focuses on "Experimental Study of A Person Identification Framework Based on Combining Left and Right Palmprint Images". 